\title{
Surface Nanopatterning by Polymer Self-Assembly: from Applied Research to Industrial Applications
}

\author{
Raphaël Pugin $^{1, *}$, Nicolas Blondiaux ${ }^{1}$, Ana Maria Popa ${ }^{1}$, Philippe Niedermann', Martha Liley ${ }^{1}$, Marta \\ Giazzon $^{1}$, Nadège Matthey ${ }^{1}$, Jeffrey Alan Hubbell ${ }^{2}$, H. Heinzelmann ${ }^{1}$ \\ 1)* Swiss Center for Eletronics and Microtechnology (CSEM SA) \\ Nanotechnology and Life Sciences Division \\ Jaquet-Droz 1, Case Postale CH-2002 Neuchatel, Switzerland, raphael.pugin@,csem.ch \\ 2) Ecole Polytechnique Fédérale de Lausanne (EPFL) \\ Laboratory for Regenerative Medicine and Pharmacobiology \\ $A A B$ 039, Station 15, CH-1015 Lausanne, Switzerland
}

\begin{abstract}
Complementary methods have been developed for surface nanostructuring using molecular self-assembly, reactive ion etching and replication techniques. First, controlled surface nanostructures with tunable lateral dimensions from tens of nanometer to tens of micrometers have been produced by polymer self-assembly, over large area and at low cost. The resulting nanostructures could find applications as antireflective layer or as security features for traceability and anti-counterfeiting applications.

Second, the as-deposited polymeric surface structures could be used as micro- and nano-patterned etch masks for the transfer of the structure into the underlying material through reactive ion etching. This process leads to more durable structures with tunable aspect ratios from 1:1 to $10: 1$ in hard materials such as silicon, silicon nitride or quartz. Potential applications of these techniques include the development of superhydrophobic and self-cleaning surfaces for MEMS, as well as the fabrication of nanoporous membranes for ultrafiltration. Polymeric nanopatterns can also be used as mastering tools for replication. Casting, embossing and moulding of self-assembled structures in plastics have all been shown to be possible.
\end{abstract}

Keywords: polymer self-assembly, nanostructures, surface nanopatterning, nanofabrication, nanoreplication, membranes

\section{Introduction}

The creation of surfaces with controlled nanoscale topographies (at least one dimensional feature below $100 \mathrm{~nm}$ ) is seen as an essential step for obtaining surfaces with radically new and unique size-dependent chemical or physical properties. The surface's optical and wetting properties as well as its ability to absorb (bio-) molecules or even whole cell are all strongly influenced by the surface topography on a nanometric scale. Thus, it is of great importance to generate surface nanostructures with a high control over their dimensions and in a flexible manner for today's application.
Standard top-down nanolithography techniques (e.g. deep UV, e-beam, Focused Ion beam) are certainly available for the fabrication of feature sizes less than $100 \mathrm{~nm}$ but remained mostly adapted to silicon-based materials using cleanroom-based fabrication processes. These increasingly expensive lithography techniques are considered for prototyping or for large-throughput manufacturing in the semiconductor industry but are not a viable manufacturing platform outside high-performance electronic [1,2]. Additionally, the broader needs in non-silicon techniques require more flexible and cost-effective massproduction methods capable of nanostructuring in 
a wide range of materials, with different length scale, and in both 2- and 3D- components.

These limitations have motivated researchers to look for new innovative and at the same time 'unconventional' methods of nanopatterning, especially for creating surface structures in the $\sim 10-150 \mathrm{~nm}$ range. These methods may represent a viable alternative if they offer advantages in reduced production cost, smaller feature sizes, or more flexibility regarding the morphology and size of nanometric structures. They would be even more attractive if suitable for the nanostructuring of 2.5D and 3D components.

Systems that show ordering and pattern formation through self-assembly may offer some of these advantages. From a technical point of view, self-assembly nanostructuring techniques are particularly promising due to their low cost, their tunability, and the increasing number of nano-building blocks that are available on the market. Among self-assembly nanopatterning processes, recent years have seen considerable progress in the development of polymer and copolymer self-assembly based nanostructured surfaces. The flexibility of these techniques (tunable parameters: polymers size, relative length of the blocks, concentration of the solution, deposition method, etc.), allows the formation of a wide range of different surface structures and makes them compatible with many existing fabrication processes. We describe in this article different approaches based on polymer selfassembly for producing highly controlled and functional nanoscale structures on surfaces. Emphasis is put on the tunability of the nanostructuring processes and on the properties of the resulting nanostructured surfaces. The use of self-assembled polymer patterns as etch mask for the transfer of the self-assembly pattern into the underlying substrate or as mastering tool for replication is also reported.

\section{Polymer self-assembly for surface nanopatterning}

\subsection{Polymer demixing}

Polymer demixing occurs when a polymer blend made of two immiscible polymers phase separates when deposited on a surface. The resulting phase separated polymeric domains are generally larger than the length of the polymer chain (micrometre, sub-micrometre range) and have peculiar morphologies (worm-like or “dots”) depending on the processing conditions. They are stochastic with respect to shape and order but present a well-defined length-scale. Various parameters can be used to tune the size and shape of the structures. Both the lateral and vertical dimensions of the domains can easily be tuned by modifying the molecular weight of the polymer, the blend concentration or the deposition parameters [1]. The morphology of the selfassembled features can be modified by adjusting the ratio between the two polymers within the blend [2]. For symmetrical blends, the system phase-separates via spinodal decomposition, leading to the formation of worm-like structures (Figure 1). In the case of very asymmetrical blends, the phase separation follows a nucleation and growth process, leading to the "dots in a matrix" pattern (Figure 2). In all cases the vertical dimension of the nanotopography obtained can be increased by removing one of the two polymers with a selective solvent.

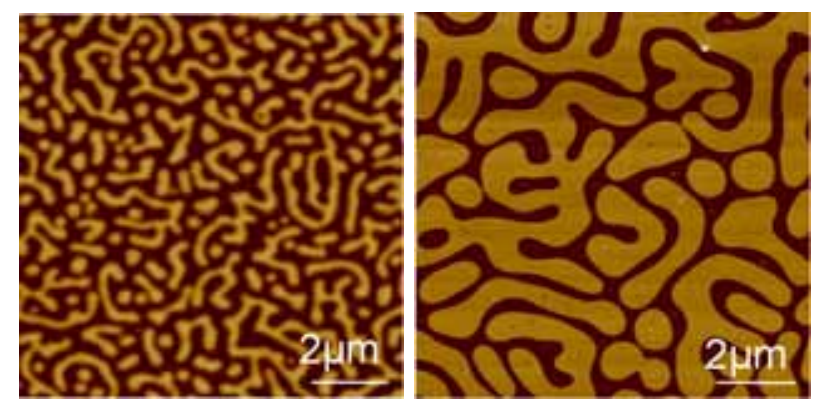

Figure 1: AFM images of phase-separated symmetrical PS/PMMA blend for different solution-concentrations (spinodal decomposition).

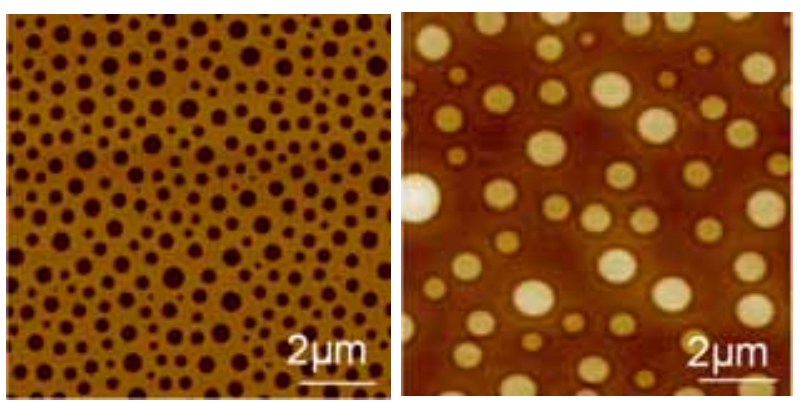

Figure 2: AFM images of phase-separated asymmetrical PS/PMMA blend for different solutionconcentrations (nucleation and growth).

Polymer demixing has already demonstrated its huge potential for a large variety of applications such as the fabrication of antireflective coatings [3], bioengineered surfaces for controlled cell growth $[4,5]$, and optimized interfaces of conjugated polymer blends for high 
performances photodiodes [6]. While the overall structure of self-assembled domains made by polymer demixing can be controlled in a flexible manner, the detailed structure remains inherently stochastic and thus irreproducible in details. Therefore we recently demonstrated the use of such unique structures as security features [7]. Similar to the characterization of true fingerprints, some key parameters can be defined to describe the self-assembled structures, which can be then be uniquely attributed to such a nanometric label. Applications in different markets, from unique identification of luxury goods to added brand protection are currently being studied.

\subsection{Block copolymer phase separation}

Similarly, phase separated domains of sub- 100 $\mathrm{nm}$ size can be formed from block-copolymers (BCs) solutions. BCs form a special class of polymers with two or more polymer chains (or blocks) covalently bound to each other. In the bulk state, BCs phase separate intermolecularly for entropic reason and the two blocks separate to a distance compatible with the size of the polymeric chains. Depending on the BC molecular weight, the volume fraction of the block, and the Flory-Huggins interaction parameter $\chi$ between the different blocks, diverse morphologies can be obtained (gyroid, cylindrical or spherical domains). When deposited on a surface using e.g. spin-coating or dip-coating, the properties of the substrate-polymer and polymerair interfaces, the substrate topography, the geometrical constraints introduced by confinement within a thin film (thickness $<100$ $\mathrm{nm}$ ) as well as the deposition parameters (e.g. spin velocity, solvent selectivity) also contribute to the morphology of the resulting BC film, in addition to the molecular characteristics of the BC. The size of the phase separated domains is on the order of the size of the macromolecules, i.e. 10$100 \mathrm{~nm}$.

An alternative strategy for the fabrication of surface nanostructures from BCs consists of the controlled deposition of highly monodisperse BC micelles preformed in solution. When dissolved in a selective solvent at a concentration higher than the critical micelle concentration, aggregation of BC molecules occurs and leads to the formation of micelles. The micelle formation and structure (size and shape) strongly depend on various parameters like e.g. concentration, block lengths, block ratios, interfacial energy between the blocks, ionic strength and solvent quality. Typically micelles can be spherical, cylindrical or wormlike.

In our earlier works we demonstrated the formation of two-dimensional arrays of polystyrene-b-poly (2-vinylpyridine) (PS-P2VP) reverse micelles on silicon surfaces [8]. The controlled tuning of the characteristics of selfassembled nanostructures has been investigated in depth. First, the periodicity of the micellar array could be systematically varied by changing BC molecular weight, concentration, spin-coating speeds and by using solvent mixture (Figure 3). Second, by varying the humidity of the spincoating environment, the film topography could be finely tuned with a high control over the height of deposited micelles.

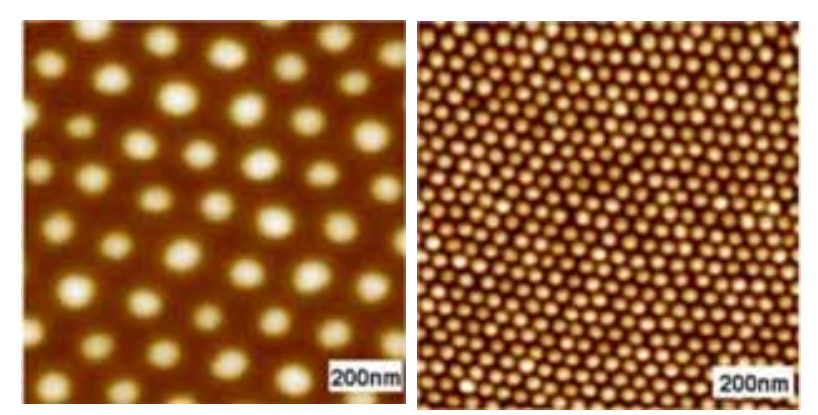

Figure 3: AFM images of arrays of BC micelles spincoated onto silicon substrates. The size and spacing of the features can be tuned by using BC with different molecular weights.

Generally the resulting nanopatterns show some short range order, however there is no longrange regularity. By guiding $\mathrm{BC}$ self-assembly onto faceted sapphire surfaces Park et al. recently described the fabrication of highly ordered ultradense arrays in BC films. The authors reported that the highly ordered pattern formed by the copolymers could be used to create hard disks with 10 terabits squeezed into a square [9].

2.3 Transfer into the underlying substrate and nanoreplication

While polymer and BC self-assembly allow the creation of a large variety of surface structures, some other functionalities can only be obtained using specific materials. Therefore self-assembled structured polymer thin films have been used as etch masks for the transfer of structures into the underlying substrate (e.g. hard materials such as Si or quartz) using standard etching processes. 
The main advantages of this approach are the fabrication of functional, more durable structures in a large variety of materials.

We recently reported the fabrication of submicrometer silicon pillars with controlled aspect ratios by combining surface structuring via polymer demixing and dry etching techniques [10]. A wide library of structures was thus achieved thanks to the tunability of both structuring processes; the lateral size of the structures was controlled via the polymer selfassembly process while the duration of dry etching provided excellent control over the height of the structures. The surfaces obtained were then rendered superhydrophobic by post depositing a low surface-energy material on the silicon pillars using either a PVD process or by means of wet silanisation. For all structures studied, water did not spread but beaded up over the surface as can be seen on the photograph inserted in Figure 4. Advancing water contact angles as high as $170^{\circ}$ could be obtained.

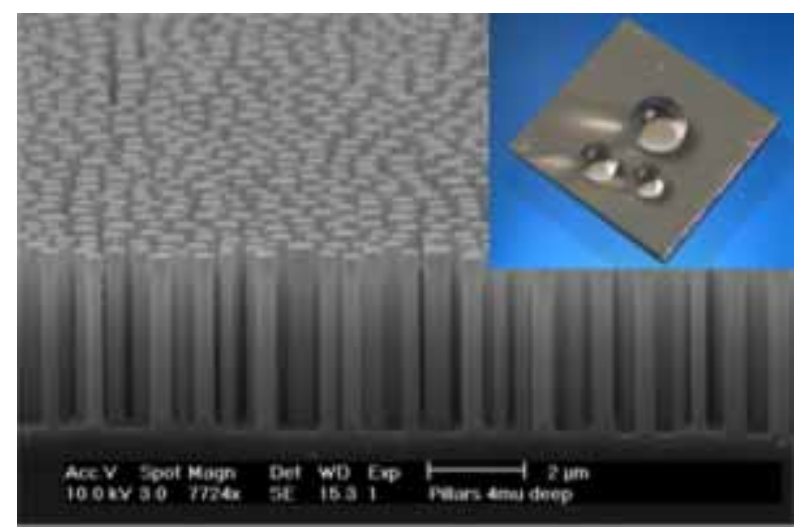

Figure 4: SEM image of high aspect ratio silicon pillars; inset: photograph of water droplet deposited on superhydrophobic surfaces (right).

Depending on the aspect-ratio of the structures, different wetting behaviours have been observed. For small aspect-ratio structures, water droplets stuck on the surfaces as indicated by the large contact angle hystereses measured. Above a 1:1 aspect-ratio, the surfaces became self-cleaning and very low contact angle hystereses, down to $5^{\circ}$, were observed.

Environmental Scanning Electron Microscopy (ESEM) was used in the wet mode in order to condense water droplets on the structured and functionalized silicon surface. As shown in Figure 5 , highly spherical water droplets were imaged on high aspect ratio pillars; no impalement of the droplet by the structures could be observed.

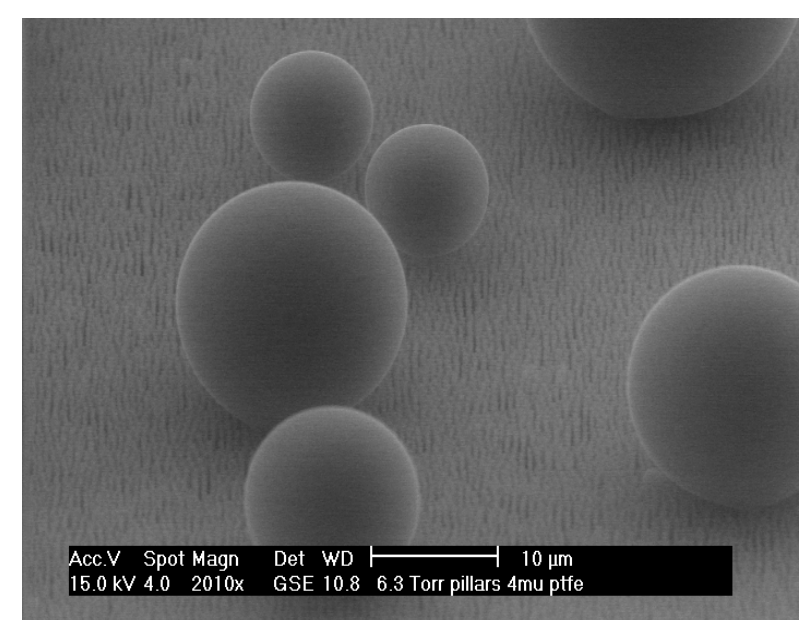

Figure 5: SEM imaged of water droplet condensed on hydrophobized high aspect ratio silicon pillars

A second application currently under investigation at CSEM is the use of structured surfaces to steer the growth and differentiation of biological cells. Numerous authors have shown that micron and nanoscale topography may change the adhesion, proliferation, morphology and differentiation of adherent cells [4,11]. We are particularly interested in the influence of surface topography on the behaviour of osteoblasts. We have shown that the morphology of SaOs-2 osteosarcoma cells depends strongly on the height of silicon pillar structures (see Figure 6). However, there is surprisingly little influence on other aspects of the cell life cycle.

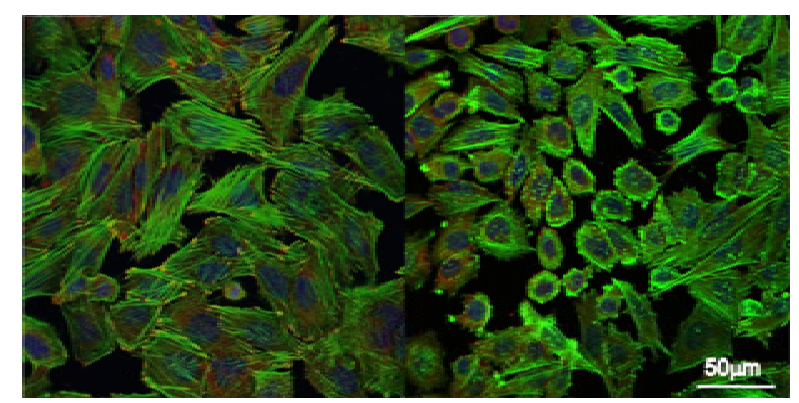

Figure 6 : Fluorescence images of SaOs-2 cells on flat (left) and structured (right) silicon surfaces. The SaOS2 cells have a rounder, less spread morphology on the structured surface. Green - actin; blue - DNA; red vinculin.

While silicon surfaces are unlikely to be suitable for use, either in Life Sciences R\&D or in applications in medical implants, the use of replication techniques may in future open the way 
to either or both of these fields. We have shown that self-assembled structures either in the form of as-deposited polymer layers, or after transfer into hard materials may be used as mastering tools for replication. Casting, embossing and moulding of self-assembled structures in plastics have all been shown to be possible.

Figure 7(left) shows a nanoporous polymeric matrix which was made by curing a PDMS prepolymer mixture onto a nanopillar structure similar the one shown in Figure 4. For 1:1 aspect ratio, the PDMS replica could be peeled off easily from the master, resulting in an elastomeric film with nanoscale holes. Similarly smaller features made by BC lithography have been replicated into TOPAS by hot embossing (Figure 7, right). In both case the periodicity of the silicon nanopillar array was preserved in the replica.
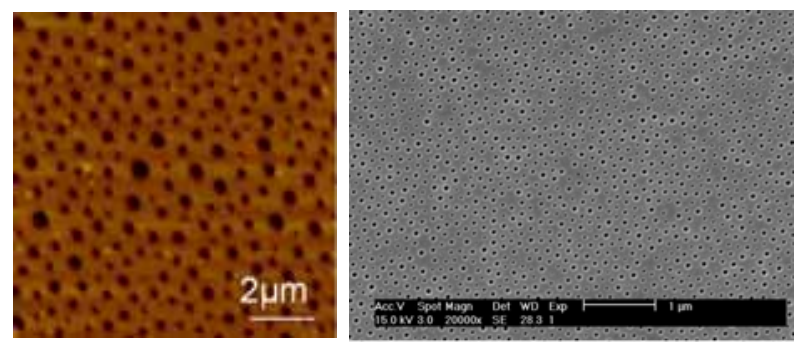

Figure 7 : AFM image of nanoporous PDMS (left) and SEM image of nanoporous TOPAS (right) obtained by replication of nanopillar structures.

These approaches could be used for the lowcost production of surface nanostructures in disposable plasticwares such as microtiter plates or cell culture flasks. Alternatively, we are currently exploring the use of surface structure in composite implant materials for orthopaedic applications.

Finally, the transfer into silicon surface of smaller structures made by BC phase separation could also be achieved. Similarly, nanopatterned BC thin films are used as etch mask during dry etch process (BC assisted lithography). Thus, CSEM has recently developed a new method for the batch fabrication of ultrathin nanoporous membrane. The proprietary process, which will soon be published, is based on the combination of BC lithography and standard microfabrication techniques and allows to produce thin silicon based membranes (thickness $<100 \mathrm{~nm}$ ) with tunable pore size ranging from 40 to $80 \mathrm{~nm}$ and a surface coverage between 12 and 25\%. Such membranes are made of silicon nitride (SiN) due to its outstanding mechanical and chemical stability; additionally they are reinforced by a supporting structure defined by photolithography in order to withstand a differential pressure of few bars (Figure 8). A $100 \mathrm{~nm}$ thick SiN layer was deposited by LPCVD on silicon wafers which were previously coated with thermally-grown silicon oxide. Using BC assisted lithography, an array of nanopores with diameters of $80 \pm 15 \mathrm{~nm}$ could then be fabricated into the SiN thin film. Standard microfabrication techniques were employed for releasing the nanoporous SiN films. Openings for membrane areas ranging from 1000 to $16000 \mu^{2}$ were defined by photolithography. Dry reactive ion etching and wet-etching were successively used for the back etch of the silicon substrate and the silicon oxide etch-stop layer.

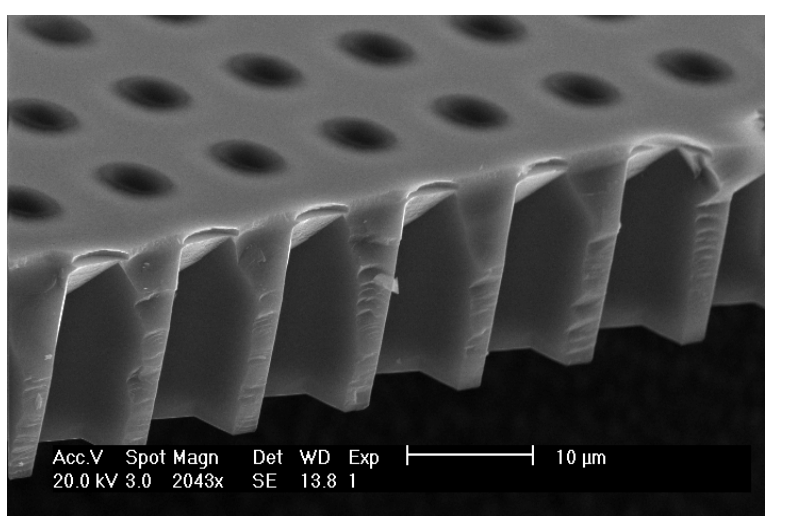

Figure 8: SEM image of the released SiN membrane. The freestanding membranes appear as dark.

In the high magnification SEM image shown in Figure 9 a high density of hexagonally packed pores with homogeneous sizes can be observed. The mean coverage of membrane surface by pore openings is $\sim 15 \%$ of the total area. Large area membranes (lateral aspect ratio thickness:width higher than 1:100) could thus be produced without any microscopic fractures or cracks.

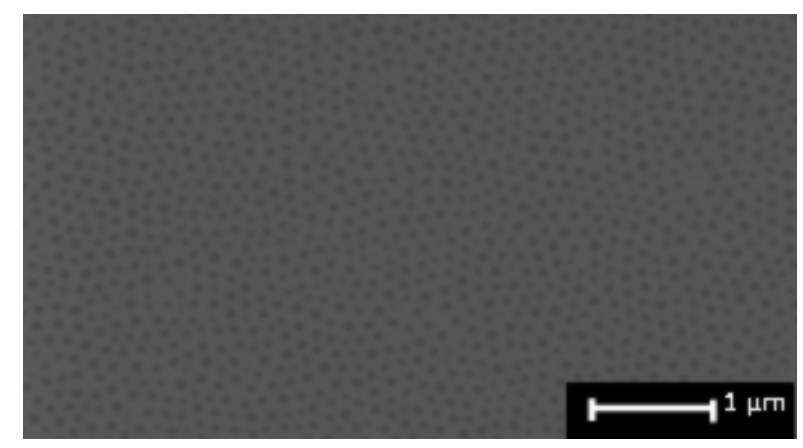

Figure 9: High magnification SEM image of the membrane back-side; the narrow pore diameter distribution is also visible. 
It was checked by FIB cross sections through the released membrane that the nanopores extend through the entire thickness of the silicon nitride film with very limited under-cut. These components are promising for applications in ultrafiltration and biosensing. First tests as well as their functionalization with stimuli responsive macromolecules for the design of smart nanovalves are currently under way.

\section{Conclusion}

Polymer and $\mathrm{BC}$ self-assembly processes are extremely promising to create surface structures on the sub-micrometer and nanometer scale. As we gain a deeper understanding of the structruring processes, we will achieve better control of feature size/morphology and resulting properties. The simplicity of this very versatile toolbox, along with its potential low-cost for generating small features over large areas, makes it very attractive for applications in various different fields such as coating or bio-technology. Additionally the general compatibility with clean room processes makes polymer self-assembly an interesting technology to integrate with standard MEMS processes, in order to obtain microsystems with new functionalities and optimized surface properties.

\section{Acknowledgments}

The work reviewed in this paper was partly funded by the State Secretariat for Education and Research, by the Swiss Nanoscience Institute (SNI) of the National Center of Competences in Research (NCCR "Nanoscale Science") and by European Union research framework program FP6. The authors thank them for their financial support. M. Dadras and M. Leboeuf are gratefully acknowledged for wet mode ESEM imaging.

\section{References}

1. M.E. Stewart, M.J. Motala, J. Yao, L.B. Thompson, and R.G. Nuzzo (2006) "Unconventional methods for forming nanopatterns" Proceedings of the Institution of Mechanical Engineers, Part J, Nanoengineering and Nanosystems, 220, (2006), 81.

2. MINAM Strategic Research Agenda (2008), http://www.minamwebportal.eu/.

3. K. Dalnoki Veress, J.A. Forrest, J.R. Stevens, J.R. Dutcher, Physica A, 239, (1997), 87.

4. K. Dalnoki Veress, J.A. Forrest, J.R. Stevens, J.R. Dutcher, Journal of Polymer Science Part BPolymer Physics, 34, (1996), 3017.

5 . S. Walheim, E. Schaffer, J. Mlynek, U. Steiner, Science, 283, (1999), 520.

6. M.J. Dalby, D. Giannaras , M.O. Riehle, N. Gadegaard, S. Affrossman, A.S.G Curtis, Biomaterials, 25, (2004), 77.

7. M. Arnold, E. Adam, R. Glass, J. Blümmel, W. Eck, M. Kantlehner, H. Kessler, J. P. Spatz, Chem Phys Chem., 5, (2004), 383.

8. E. Moons, Journal of Physics-Condensed Matter, 14, (2002), 12235.

9. H. Heinzelmann, N. Blondiaux, A.M. Popa, T. Overstolz, A. Hoogerwerf, M. Giazzon, G.

Weder, M. Liley, R. Pugin, European Coating Journal, 4, (2009), 132.

10. S. Krishnamoorthy, R. Pugin, J. Brugger, H. Heinzelmann, C. Hinderling, Adv. Funct. Mater., 16, (2006), 1469.

11. S. Park, D.H. Lee, J. Xu, B. Kim, S.W. Hong, U. Jeong, T. Xu, T.P. Russell, Science, 323, (2009), 1030.

12 . N. Blondiaux, A.M. Popa, E. Scolan, J. Gavillet, R. Pugin, Applied Surface Science, in press.

13. J.O. Gallagher, K.F. McGhee, C.D.W. Wilkinson, M.O. Riehle, IEEE Trans Nanobiosci., 1, (2002), 24. 